\title{
Predictions of the vapor-liquid equilibrium data for low-GWP Hydrofluorocarbons + polyethylene-glycol dimethylether solvents by modified UNIFAC model
}

\author{
Xiao Zhang ${ }^{1}$, Liang Cai ${ }^{1,}{ }^{*}$, Jingyi Qiao ${ }^{1}$ \\ ${ }^{1}$ School of Energy and Environment, Southeast University, Nanjing 210096, P R China;
}

\begin{abstract}
The working fluids are crucial for developing the absorption refrigeration system. Our previous work has proposed three novel working pairs, which uses the low-GWP HFCs (R32, R152a and R161) as the refrigerants and the polyethylene-glycol dimethylether solvent DMETEG as the absorbent. To explore the VLE behaviors of HFCs in other longer chain structures of polyethylene-glycol dimethylether solvents, the modified UNIFAC (Dortmund) model was applied for prediction. The previous binary parameters showed large deviations from the experimental data. Then the new binary parameters were obtained by regression. For R32+DMEDEG and R152a/R161+DMETrEG, the prediction ARAD results were reduced from 17.13\%, $24.06 \%$ and $12.48 \%$ to $3.71 \%, 2.61 \%$ and $6.86 \%$, respectively. Finally, the VLE data of R161 + PGDE at the temperatures of $293.15-343.15 \mathrm{~K}$ were predicted.
\end{abstract}

\section{Introduction}

The absorption refrigeration system is of great significance for the energy utilization, especially the waste heat source. It can greatly promote my country's process of achieving carbon peaking or carbon neutrality. The working pairs of the absorption system is crucial as they greatly influence the performances of the system. Thus, a lot of researchers have recently explored the novel binary working pairs using the low-GWP Hydrofluorocarbons (HFCs, i.e., R32, R152a, and R161) as the refrigerant and the organic solvents as the absorbent.

Deng et al. [1] developed the three binary working fluids, R161+DMF/DMEDEG and R134a+DMF. their vapor-liquid equilibrium (VLE) data were measured by the static-analytical method. The results showed that the R161+DMEDEG is more favorable for the hybrid refrigeration system. Also three new binary mixtures were compared by Li et al. [2] based on the five-parameter nonrandom two-liquid (NRTL) activity coefficient model. From low to high, the solubilities are arranged into $\mathrm{R} 152 \mathrm{a}+\mathrm{DMEDEG}<\mathrm{R} 32+\mathrm{DMAC}<\mathrm{R} 32+\mathrm{DMEDEG}$. The VLE data of R161 + DMAC/NMP at a temperature of 293.15-353.15K were also measured and correlated by Jing et al[3]. Besides, because of the atmospheric lifetime of only 11 days and the 100-year GWP about 4 [4], the extremely environmentally friendly R1234yf was explored by Fang et al. [5] for its solubility in NMP and DMETrEG by using a dual-cycle experimental device.

Our previous work [6] has proved the strong affinity of polyethylene-glycol dimethylether solvents for the low GWP HFCs, it's vital to explore the influences of longer chain structures of ether solvents on the VLE behaviors of the HFCs refrigerants. However, it's unrealistic to perform each VLE measurement. The group contribution method, modified UNIFAC (Dortmund) model, is a good theory to predict the VLE behaviors of the solutions. So, in this work, the new interaction parameters of the modified UNIFAC model are obtained for the VLE predictions of the low GWP HFCs+ polyethylene-glycol dimethylether mixtures.

\section{Methodology}

The eight different reported VLE data [1, 2, 6-8] were used for reaches. For any binary system, its inner equilibrium states can be expressed as:

$$
p=p_{1}^{s} x_{1} \gamma_{1} \exp \left(\frac{\left(V_{1}^{s l}-B_{1}\right)\left(p-p_{1}^{S}\right)}{R T}\right)
$$

Where $p, T$ are the system pressure and temperature, respectively; $x_{1}$ is the refrigerant mole fraction; $p_{1}^{s}, V_{1}^{s l}$ and $B_{1}$ are the saturated vapor pressure, saturated mole volume of liquid refrigerants and second virial coefficient at the temperature $T$, respectively, which can be obtained from REFPROP 9.1; $\gamma_{1}$ is the refrigerant activity coefficient and is calculated by the modified UNIFAC (Dortmund) model.

The modified UNIFAC model is developed based on the original UNIFAC model. The activity coefficient of the species $i$ is the function of temperature and composition of the solution, and can be expressed as the sum of a combinatorial and a residual part:

$$
\ln \gamma_{1}=\ln \gamma_{i}^{C}+\ln \gamma_{i}^{R}
$$

The combinatorial part $\ln \gamma_{i}^{C}$ reflects the repulsive interactions caused by the different molecular groups' size

* Corresponding author: Cailiang@seu.edu.cn 
and shape, and is treated by the empirical way:

$$
\begin{gathered}
\ln \gamma_{i}^{C}=1-V_{i}^{\prime}+\ln V_{i}^{\prime}-\frac{Z}{2} q_{i}\left(1-\frac{V_{i}}{F_{i}}+\ln \left(\frac{V_{i}}{F_{i}}\right)\right) \\
V_{i}^{\prime}=\frac{r_{i}^{3 / 4}}{\sum_{j} x_{j} r_{j}^{3 / 4}} ; F_{i}=\frac{q_{i}}{\sum_{j} x_{j} q_{j}} ; V_{i}=\frac{r_{i}}{\sum_{j} x_{j} r_{j}}
\end{gathered}
$$

Where $\mathrm{Z}$ is commonly equal to $10 ; r_{i}$ and $q_{i}$ are the relative van der Waals volumes and surface area, respectively. $r_{i}$ and $q_{i}$ can be calculated from:

$$
r_{i}=\sum_{k} v_{k}^{(i)} R_{k} ; q_{i}=\sum_{k} v_{k}^{(i)} Q_{k}
$$

Where $v_{k}^{(i)}$ stands for the number of molecular group $k$ of specie $i ; R_{k}$ and $Q_{k}$ are the volume parameter and surface area parameter of group $k$, respectively.

The residual activity coefficient, $\ln \gamma_{i}^{R}$, reflects the intermolecular interactions attributed to the different molecular groups. It is the sum of group activity coefficients deviations between the solution and pure component, as expressed by:

$$
\ln \gamma_{i}^{R}=\sum_{k} v_{k}^{(i)}\left(\ln \Gamma_{k}-\ln \Gamma_{k}^{(i)}\right)
$$

Where $\Gamma_{k}$ and $\Gamma_{k}^{(i)}$ stand for the activity coefficient of group $k$ in solution and pure component $i$, respectively. They can be obtained as follows:

$$
\begin{gathered}
\ln \Gamma_{k}=Q_{k}\left[1-\ln \left(\sum_{m} \theta_{m} \Psi_{m k}\right)-\sum_{m}\left(\theta_{m} \Psi_{k m} / \sum_{n} \theta_{n} \Psi_{n m}\right)\right] \\
\theta_{m}=\frac{Q_{m} X_{m}}{\sum_{n} Q_{n} X_{n}} ; X_{m}=\frac{\sum_{i} v_{m}^{(i)} x_{i}}{\sum_{i} \sum_{k} v_{k}^{(i)} x_{i}}
\end{gathered}
$$

Where $\theta_{m}$ and $X_{m}$ denote the surface area fraction and the mole fraction of group $m$ in solution; $\Psi_{m n}$ denotes the time-dependent group interaction parameter between groups $m$ and $n$, as expressed by:

$$
\Psi_{m n}=\exp \left(-\frac{a_{m n}+b_{m n} T+c_{m n} T^{2}}{T}\right)
$$

Where $a_{m n}(\mathrm{~K}), b_{m n}$ and $c_{m n}\left(\mathrm{~K}^{-1}\right)$ are the adjustable interaction parameters regressed from the corresponding experimental data. It's noted that for the calculation of $\Gamma_{k}^{(i)}, \theta_{m}$ and $X_{m}$, they are treated in pure component $i$.
Because of the strong electronegativity effects caused by the strongly polarizing atoms (i.e. $\mathrm{F}, \mathrm{Cl}$ or $\mathrm{O}$ ) in $\mathrm{HFCs}$, Kleiber and Axmann proposed the new group assignments and extended ten additional structure groups to the modified UNIFAC models to reduce the vapor-liquid prediction deviations of the mixtures containing the common fluorinated refrigerants. The corresponding volume parameters $\left(R_{k}\right)$, surface area parameters $\left(Q_{k}\right)$ and temperature-dependent interaction parameters of the new structure groups were determined through regressing the VLE experimental database. Thus, the calculation results of their previously correlated group interaction parameters are used for comparison.

According to the new group assignments proposed by Kleiber and Axmann [9, 10], for the halogenated methane derivatives, i.e. $\mathrm{CH}_{\mathrm{m}} \mathrm{F}_{\mathrm{n}}(\mathrm{m}+\mathrm{n}=4)$, the different main groups were defined based on its number of $\mathrm{F}$ atoms, i.e. $\mathrm{CHF}, \mathrm{CHF}_{2}$ and $\mathrm{CHF}_{3}$; while for the halogenated ethane derivatives with a $\mathrm{CH}_{3}$ segment, i.e. $\mathrm{CH}_{3} \mathrm{CH}_{m} \mathrm{~F}_{n}(\mathrm{~m}+\mathrm{n}=3)$, the $\mathrm{CH}_{3}$ segment can be replaced by an $\mathrm{H}$ atom and the ethane derivative yields the aforementioned corresponding methane derivatives, so the halogenated ethane derivatives can be divided into a $\mathrm{CHF}_{\mathrm{n}}$ group and a $\mathrm{CH}_{3}$ group. Then the HFCs refrigerants, R32, R152a and $\mathrm{R} 161$, are segmented into the corresponding groups as listed in 0 . Because of the same structure formula, $\mathrm{H}_{3} \mathrm{C}\left[\mathrm{OCH}_{2} \mathrm{CH}_{2}\right]_{n} \mathrm{OCH}_{3}$, the polyethylene-glycol dimethylether chemical solvents have four different molecular groups $\left(\mathrm{CH}_{3}, \mathrm{CH}_{2}, \mathrm{CH}_{2} \mathrm{O}, \mathrm{CH}_{3} \mathrm{O}\right)$. Several different functional-group assignment configurations were proposed. And the configuration consists of $2 \mathrm{CH}_{3}$ groups, $n-1 \mathrm{CH}_{2}$ groups and $n+1 \mathrm{CH}_{2} \mathrm{O}$ groups were found the lowest deviations when used for the regressions among all the configurations. Therefore, this configuration is adopted in this work and the group assignments are also listed in 0 . The relevant volume parameters and the surface area parameters of the subgroups are obtained from Kleiber and Axmann and Gmehling et al.[11]. The group interaction parameters between main groups taken from Kleiber, Axmann and Gmehling et al. [11] are used as the previous parameters for the result comparisons.

Table 1. Modified UNIFAC Group assignments for the HFCs refrigerants and the chemical solvents

\begin{tabular}{cccccc}
\hline Substances & Subgroups & Main groups & No. & $R_{k}$ & $Q_{k}$ \\
\hline \multirow{2}{*}{ R32 } & $\mathrm{CH}_{2} \mathrm{~F}_{2}$ & $\mathrm{CHF}_{2}$ & 1 & 1.7529 & 1.8147 \\
R152a & $\mathrm{CHF}_{2}$ & $\mathrm{CHF}_{2}$ & 1 & 1.1396 & 1.439 \\
& $\mathrm{CH}_{3}$ & $\mathrm{CH}_{2}$ & 1 & 0.6325 & 1.0608 \\
R161 & $\mathrm{CH}_{2} \mathrm{~F}$ & $\mathrm{CHF}$ & 1 & 1.0685 & 1.0007 \\
& $\mathrm{CH}_{3}$ & $\mathrm{CH}_{2}$ & 1 & 0.6325 & 1.0608 \\
DMEDEG & $\mathrm{CH}_{3}$ & $\mathrm{CH}_{2}$ & 2 & 0.6325 & 1.0608 \\
& $\mathrm{CH}_{2}$ & $\mathrm{CH}_{2}$ & 1 & 0.6325 & 0.7081 \\
& $\mathrm{CH}_{2} \mathrm{O}$ & $\mathrm{CH}_{2} \mathrm{O}$ & 3 & 1.1434 & 1.2495 \\
DMETrEG & $\mathrm{CH}_{3}$ & $\mathrm{CH}_{2}$ & 2 & 0.6325 & 1.0608 \\
& $\mathrm{CH}_{2}$ & $\mathrm{CH}_{2}$ & 2 & 0.6325 & 0.7081 \\
& $\mathrm{CH}_{2} \mathrm{O}$ & $\mathrm{CH}_{2} \mathrm{O}$ & 4 & 1.1434 & 1.2495 \\
DMETEG & $\mathrm{CH}_{3}$ & $\mathrm{CH}_{2}$ & 2 & 0.6325 & 1.0608 \\
& $\mathrm{CH}_{2}$ & $\mathrm{CH}_{2}$ & 3 & 0.6325 & 0.7081 \\
& $\mathrm{CH}_{2} \mathrm{O}$ & $\mathrm{CH}_{2} \mathrm{O}$ & 5 & 1.1434 & 1.2495 \\
\hline
\end{tabular}




\section{Results and discussion}

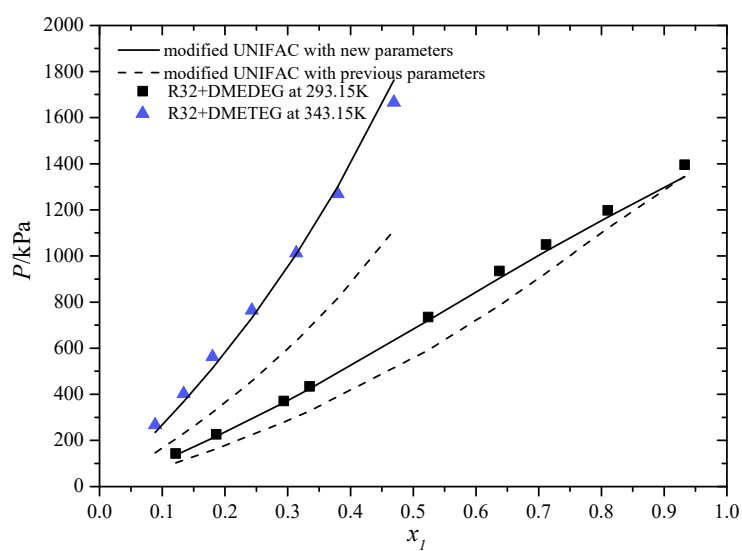

(a) R32

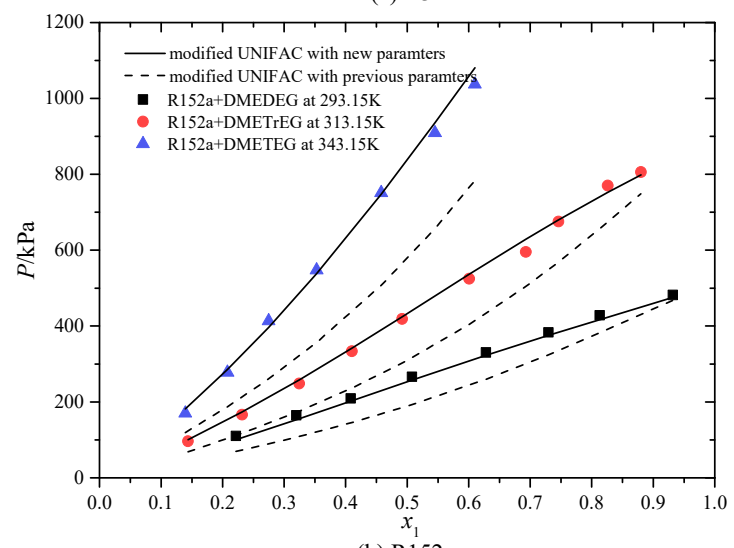

(b) R152a

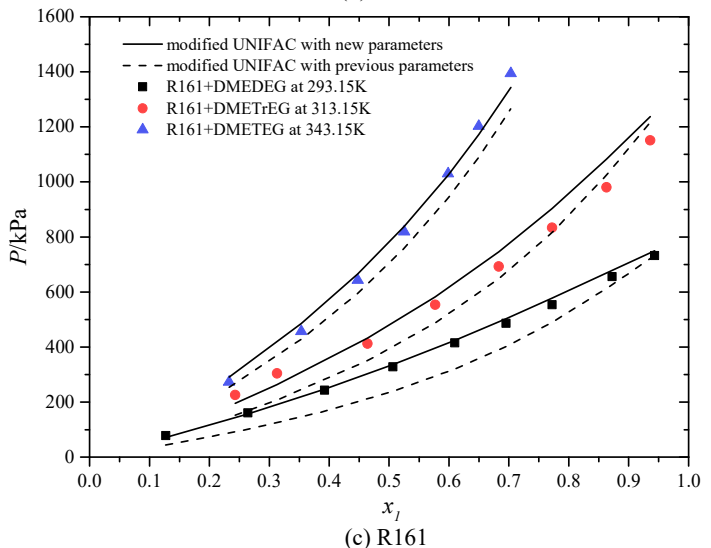

Figure 1. Experimental and calculated vapor pressures of R32/R152a/R161 in several polyethylene-glycol dimethylether solvents at different temperatures; symbols: the experimental data; solid line: modified UNIFAC with new parameters; dashed line: modified UNIFAC with previous parameters
In this study, the new group interaction parameters of the modified UNIFAC (Dortmund) model are obtained by regressing the experimental VLE data. Considering the group assignments of the HFCs, the five binary mixtures, R32+DMETEG, R152a+DMEDEG/DMETEG and R161+DMEDEG/DMETEG, are selected for the regression. And the other measured VLE data of R32+DMEDEG, R152a/R161+DMETrEG are used for verification. This regression is carried out by the leastsquares method using the MATLAB software. Eq. (1) is applied to describe the non-ideality VLE behavior of the binary mixtures and the maximum-likelihood principle is used as the objection function for minimization. 0 shows the regressed new group interaction parameters of the modified UNIFAC model. 0 illustrates the experimental and calculated vapor pressures by the previous and new interaction parameters of the studied $8 \mathrm{HFCs}+$ polyethylene-glycol dimethylether binary mixtures at the temperatures of $293.15,313.15$ and $343.15 \mathrm{~K}$, respectively.

For the five binary mixtures used for regression, the average relative absolute deviation (ARAD) between the experimental and calculated pressures using the new parameters is about $4.37 \%$, while the modified UNIFAC (Dortmund) model using the previous parameters shows an ARAD of $18.69 \%$ from the experimental data. The regressed results are found in better agreement with the experimental data as depicted in 0 . Also, for the remaining three mixtures, R32+DMEDEG and $\mathrm{R} 152 \mathrm{a} / \mathrm{R} 161+\mathrm{DMETrEG}$, the prediction ARAD calculated by the regressed new parameters are $3.71 \%$, $2.61 \%$ and $6.86 \%$; while the prediction ARAD calculated by the previous parameters shows $17.13 \%, 24.06 \%$ and $12.48 \%$, respectively. All the results prove that the new regressed parameters of the modified UNIFAC model have lower prediction deviations than that obtained from literature, and they can be used to predict the isothermal solubilities of HFCs + polyethylene-glycol dimethylether binary mixtures. Finally, the predicted VLE data of R161 + PGDE (Pentaethyleneglycol dimethyl ether, CAS:119187-3, $\left.\mathrm{H}_{3} \mathrm{C}\left[\mathrm{OCH}_{2} \mathrm{CH}_{2}\right]_{5} \mathrm{OCH}_{3}\right)$ at the temperatures of $293.15-343.15 \mathrm{~K}$ are illustrated in 0.

Table 2. New group interaction parameters of modified UNIFAC (Dortmund)

\begin{tabular}{cccccccc}
\hline Main group $m$ & Main group $n$ & $a_{m n} / \mathrm{K}$ & $a_{n m} / \mathrm{K}$ & $b_{m n}$ & $b_{n m}$ & $c_{m n} / \mathrm{K}^{-1}$ & $c_{n m} / \mathrm{K}^{-1}$ \\
\hline $\mathrm{CHF}_{2}$ & $\mathrm{CH}_{2}$ & -0.0539 & -0.2103 & 2.96044 & -2.4577 & -0.0083 & 0.00721 \\
$\mathrm{CHF}_{2}$ & $\mathrm{CH}_{2} \mathrm{O}$ & 0.69702 & 0.9682 & 2.59157 & -2.3053 & -0.006 & 0.00608 \\
$\mathrm{CHF}$ & $\mathrm{CH}_{2}$ & 0.00111 & -0.0161 & -0.2944 & 13.7253 & -0.00036 & -0.0514 \\
$\mathrm{CHF}$ & $\mathrm{CH}_{2} \mathrm{O}$ & 0.10316 & 0.19416 & -5.7566 & 14.5816 & 0.02258 & -0.0543 \\
\hline
\end{tabular}




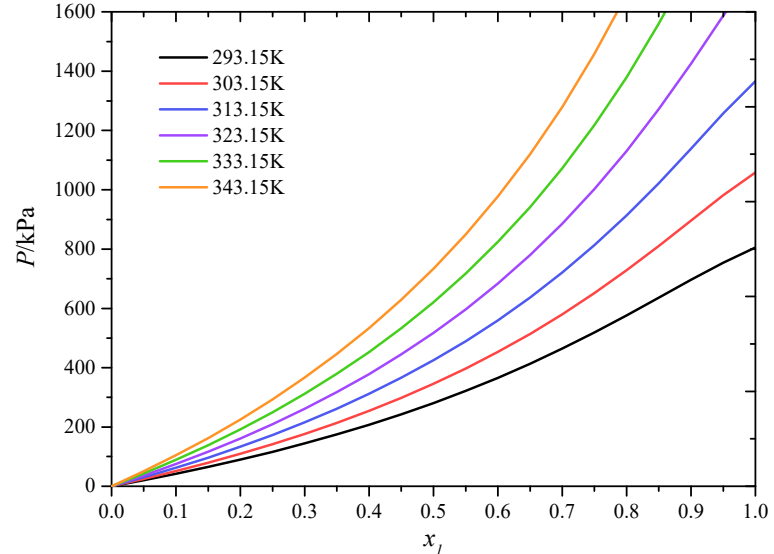

Figure 2. $p$-T- $x$ diagram for the predicted VLE results of R161(1) + PGDE (2) by modified UNIFAC model with new parameters

\section{Conclusions}

An advanced group contribution model based on the molecular structure, the modified UNIFAC (Dortmund) model, was introduced to predict the VLE behaviors of HFCs + polyethylene-glycol dimethylether chemical solvents when lacking of experimental data.

The VLE data of all reported eight working fluids were studied, of which five binary mixtures were adopted for regression and the other three were used for prediction verification. The results showed that our regressed model had an average regression deviation of $4.37 \%$ while the modified UNIFAC model using the previous parameters showed a deviation of $18.69 \%$. As for their prediction, our model also showed the lower deviations than that of the reference.

Finally, the VLE data of R161 + PGDE (Pentaethyleneglycol dimethyl ether, CAS:1191-87-3, $\mathrm{H}_{3} \mathrm{C}\left[\mathrm{OCH}_{2} \mathrm{CH}_{2}\right]_{5} \mathrm{OCH}_{3}$ ) at the temperatures of 293.15$343.15 \mathrm{~K}$ were predicted by our regressed model.

\section{Acknowledges}

This work was financed by the National Key Research and Development Program of China (Grant No. 2018YFC0705306), the Natural Science Foundation of China (Grant No. 6503000103).

\section{References:}

1. Deng, R., et al., Vapor - liquid equilibrium measurements and assessments of fluoroethane $+\mathrm{N}, \mathrm{N}$-dimethylformamide and fluoroethane + dimethylether diethylene glycol systems for the hybrid refrigeration cycle. International Journal of Refrigeration, 2014. 43: p. 176-186.

2. Li, X., et al., Vapor - liquid equilibria of difluoromethane $+\mathrm{N}, \mathrm{N}$-dimethylacetamide, difluoromethane+dimethylether diethylene glycol and 1,1-difluoroethane+dimethylether diethylene glycol systems. Fluid Phase Equilibria, 2013. 347: p.
$15-21$.

3. Jing, X., R. Deng and D. Zheng, Measurement and Correlation of Isothermal Vapor - Liquid Equilibrium of Fluoroethane $+\mathrm{N}$, NDimethylacetamide, Fluoroethane +N-Methyl-2pyrrolidone, and 1,1,1,2-Tetrafluoroethane +N,NDimethylacetamide Systems. Journal of Chemical \& Engineering Data, 2013. 58(11): p. 3289-3296.

4. Wu, W., et al., Comparative analysis of conventional and low-GWP refrigerants with ionic liquid used for compression-assisted absorption cooling cycles. Applied Thermal Engineering, 2020. 172: p. 115145.

5. Fang, Y., et al., Isothermal Vapor - Liquid Equilibria of the Absorption Working Pairs (R1234yf + NMP, R1234yf + DMETrEG) at Temperatures from 293.15 $\mathrm{K}$ to $353.15 \mathrm{~K}$. Journal of Chemical \& Engineering Data, 2018. 63(5): p. 1212-1219.

6. Zhang, X., et al., Vapor-liquid equilibrium measurements and assessments of Low-GWP absorption working pairs (R32+DMETEG, R152a+DMETEG, and R161+DMETEG) for absorption refrigeration systems. Energy, 2021. 224: p. 120082.

7. Feng, L., et al., Measurement and Correlation of Isothermal Vapor - Liquid Equilibrium of Fluoroethane + Dimethyl Ether Triethylene Glycol, 1,1-Difluoroethane + Dimethyl Ether Triethylene Glycol, and 1,1-Difluoroethane + N - Methyl-2pyrrolidone Systems. 2016.

8. Han, X., et al., Isothermal Vapor - Liquid Equilibrium of HFC-161 + DMETrEG within the Temperature Range of 293.15 - $353.15 \mathrm{~K}$ and Comparison for HFC-161 Combined with Different Absorbents. Journal of Chemical \& Engineering Data, 2016. 61(3): p. 1321-1327.

9. Kleiber, M. and J.K. Axmann, Evolutionary algorithms for the optimization of Modified UNIFAC parameters. Computers and Chemical Engineering, 1998. 23(1): p. 63-82.

10. Kleiber, M., An extension to the UNIFAC group assignment for prediction of vapor-liquid equilibria of mixtures containing refrigerants. Fluid Phase Equilibria, 1995. 107(2): p. 161-188.

11. Gmehling, J., J. Li and M. Schiller, A modified UNIFAC model. 2. Present parameter matrix and results for different thermodynamic properties. Industrial \& Engineering Chemistry Research, 1993. 32(1): p. 178-193. 